LA GRANA:

REVISTA DE

CIENCIAS DE LA VIDA

pISSN:1390-3799; eISSN:1390-8596

http:/ / doi.org/10.17163/lgr.n30.2019.06
Artículo científico / Scientific paper

RIESGOS NATURALES

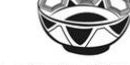

ABYA UNIVERSIDAD YALA POLITECNICA

\title{
REGIONALIZACIÓN DE LA PRECIPITACIÓN, SU AGRESIVIDAD Y CONCENTRACIÓN EN LA CUENCA DEL RÍO GUAYAS, ECUADOR
}

\author{
REGIONALIZATION OF PRECIPITATION, ITS AGGRESSIVENESS AND \\ CONCENTRATION IN THE GUAYAS RIVER BASIN, ECUADOR
}

\author{
Mercy Ilbay-Yupa*1 ${ }^{*}$, Ricardo Zubieta Barragán ${ }^{2}$ () y Waldo \\ Lavado-Casimiro ${ }^{3}$ ()
}

\author{
${ }^{1}$ Facultad de Ciencias Agropecuarias y Recursos Naturales, Universidad Técnica de Cotopaxi, Av Simón Rodríguez, Latacunga, \\ Ecuador \\ 2 Departamento de Ciencias de la Atmósfera e Hidrósfera, Instituto Geofísico del Perú, Av. Badajoz 169, Ate 15012, Lima, Perú \\ 3 Programa de Doctorado en Recursos Hídricos, Universidad Nacional Agraria La Molina, La Molina 15024 Lima, Perú \\ *Autor para correspondencia: mercy.ilbay@utc.edu.ec
}

\begin{abstract}
Resumen
La agresividad de la lluvia contribuye a la erosividad del suelo en regiones de alta montaña, y por ende a la sedimentación en la parte baja de la cuenca. El conocimiento acerca de la agresividad de la lluvia en regiones costeras y andinas contribuye a la formulación de medidas de mitigación que influyen en la reducción de erosión y pérdida de nutrientes. Los índices Fournier, Fournier modificado y de concentración de precipitación proveen la capacidad de estimar la distribución espacial y temporal de la agresividad de la lluvia. Este estudio presenta un análisis de la lluvia mediante estos índices de agresividad en la cuenca del río Guayas ubicada en la costa y Andes ecuatoriales. Se seleccionaron datos mensuales registrados de 30 estaciones pluviométricas para el período 1968-2014. Se determinaron zonas homogéneas de precipitación mediante el método k-means. Los resultados indicaron dos regiones homogeneas predominantes, la primera ubicada al oeste en la zona costera y andina (85,2\% del área de la cuenca), con un índice de agresividad alto y muy alto; mientras que la distribución de la precipitación en la segunda región (Alta montaña) resultó de muy baja a baja agresividad. La mayor agresividad potencial de la lluvia le corresponde una mayor acumulación de precipitación promedio anual, lo que indica una alta influencia estacional de las lluvias, es decir, una mayor cantidad de lluvia puede precipitar en un número reducido de meses consecutivos. Los valores de concentración revelan un gradiente regional en dirección este-oeste que va de moderadamente a fuertemente estacional. El análisis de tendencias de la concentración de lluvia mensual no muestra cambios significativos en el período de estudio. No obstante, los hallazgos del presente estudio explican el porqué la región oeste y sur de la cuenca del río Guayas está expuesta a problemas de sedimentación en la parte baja, producto de la capacidad erosiva de la lluvia en la parte alta y media de la cuenca.
\end{abstract}

Palabras clave: Guayas, concentración, precipitación, agresividad, erosividad, Ecuador. 


\begin{abstract}
The aggressiveness of rain contributes to the erosion of the soil in high mountain regions, and therefore to the sedimentation in the lower part of the watershed. To know about the aggressiveness of rain in coastal and Andean regions contributes to the formulation of mitigation measures that help to the reduction of erosion and loss of nutrients. Fournier indices, Modified Fournier and precipitation concentration provide the ability to estimate the spatial and temporal distribution of the aggressiveness of the rain. This study presents a spatial and temporal analysis of climatic aggressiveness in the Guayas river watershed located on the coast and the equatorial Andes. Registered monthly data of 30 rainfall stations for the period 1968-2014 was selected. Homogeneous precipitation zones were determined by the k-means method. The results indicated two predominant homogenous regions, the first located to the west in the coastal and Andean zone (85,2\% of the area of the Watershed), with a high and very high aggressiveness index, while the distribution of precipitation in the second region (High mountain) resulted from very low to low aggressiveness. The greater potential aggressiveness of rain corresponds to a greater accumulation of average annual rainfall, which indicates a high seasonal influence of rainfall, i.e., a greater amount of rainfall can precipitate in a reduced number of consecutive months. The concentration values reveal a regional gradient in the east-west direction, which goes from moderately to strongly seasonal. The trend analysis of the monthly rainfall concentration shows no significant changes in the study period. However, these findings explain why the western and southern region of the Guayas river watershed is exposed to sedimentation problems in the lower part, due to the erosive capacity of rain in the higher and middle part of the watershed.
\end{abstract}

Keywords: Guayas, concentration, precipitation, aggressiveness, erosivity, Ecuador.

Forma sugerida de citar: Ilbay-Yupa, M., Zubieta B., R. y Lavado-Casimiro, W. (2019). Regionalización de la precipitación, su agresividad y concentración en la Cuenca del río Guayas, Ecuador. La Granja: Revista de Ciencias de la Vida. Vol. 30(2):57-76. http://doi.org/10.17163/lgr. n30.2019.06.

IDs Orcid:

Mercy Ilbay-Yupa: http:/ / orcid.org/0000-0001-9503-2686

Ricardo Zubieta Barragán: https:/ / orcid.org/0000-0002-4315-7695

Waldo Lavado-Casimiro: https:/ / orcid.org/0000-0002-0051-0743 


\section{Introducción}

La erosividad de las precipitaciones provoca pérdidas de suelo fértil, daños a la infraestructura, agricultura y contaminación del agua, la cual está influenciada por los cambios en los patrones de precipitación (Martín-Fernández y Martínez-Núñez, 2011; Sanchez-Moreno, Mannaerts y Jetten, 2014); esto ocurre ya que grandes cantidades de lluvia estacional puede precipitar en muy pocos días o semanas en regiones de alta montaña como los Andes (Sarricolea, Araya y Salazar, 2014; Zubieta y col., 2016; Sarricolea y col., 2019), o regiones Amazónicas (Zubieta y col., 2019). La precipitación es una variable importante para estudios climáticos, cuya variabilidad espacial y temporal pueden impactar en las actividades humanas durante eventos hidroclimáticos extremos como sequías e inundaciones (Parracho, Melo-Gonçalves y Rocha, 2016). Además desempeña un papel crucial en la planificación y gestión de los recursos hídricos, vinculada directamente con la agricultura y la mitigación de desastres (Prakash y col., 2015). La cuantificación exacta de la precipitación sigue siendo un reto para muchas aplicaciones hidrológicas, especialmente en regiones con topografía compleja debido a efectos orográficos y pendiente en pequeña escala (Sevruk, 2004; Zubieta y col., 2015).

La agresividad de la lluvia puede causar impactos ambientales y es un factor clave para la ocurrencia de erosión de suelos, deslizamientos o inundaciones. Por lo tanto, los parámetros que evalúen la agresividad de la lluvia pueden ser considerados como un indicador ambiental apropiado (Gregori, Andrenelli y Zorn, 2006; García-Barrón, Morales y Sousa, 2018). La estimación de esta variable durante largos períodos es útil para la conservación del suelo, la planificación agrícola y el desarrollo de políticas ambientales. El factor R o erosividad de la lluvia es un instrumento aceptado para la medición de la erosión local y depende de la energía cinética de cada episodio de lluvia (Panagos y col., 2015). Este modelo es el más utilizado y fue desarrollado a una escala detallada en el sector agrícola; sin embargo, su aplicación a escala regional presenta limitaciones (Terranova y col., 2009). Las estimaciones de la erosión del suelo no se ajustan a las medidas empíricas de sedimentación y no incorpora el escurrimiento directo del agua (Kinnell, 2010). El factor $\mathrm{R}$ sobreestima la erosión a nivel regional o de cuen- ca hidrográfica (Hernando y Romana, 2016) y no se recomienda en áreas donde no se realiza un proceso de validación. Además, para su estimación es conveniente utilizar registros de precipitaciones de alta frecuencia de estaciones meteorológicas durante un período superior a veinte años (Angulo-Martínez y col., 2009).

Por ello, se pueden utilizar modelos que miden los efectos de la agresividad de la lluvia, considerando registros de precipitaciones por hora (modelos de intensidad) o registros de precipitaciones mensuales (modelos de volumen). Este último modelo se refiere a las diferentes acumulaciones parciales de lluvia, sin tener en cuenta el número, la duración y la cantidad de lluvia de cada episodio, por lo que se basa exclusivamente en registros mensuales de precipitaciones disponibles en la mayoría de los países. Así, se puede utilizar el índice de agresividad en estudios ambientales (Fournier, 1960; Arnoldus, 1978; Oliver, 1980). Estos índices han sido ampliamente utilizados en estudios climáticos para identificar patrones espaciales de concentración de lluvia en regiones de Europa, Asia, África y Sudamérica tales como España (De Luis y col., 2011), Península Ibérica (García-Barrón, Morales y Sousa, 2018), India (Ballari y col., 2018), Bangladesh (Rasel, Islam y Keramat, 2016), Nigeria (Ezenwaji, Nzoiwu y Chima, 2017), Argentina (Besteiro y Delgado, 2011), Venezuela (Rey y col., 2012) y Chile (Sarricolea, Araya y Salazar, 2014; Valdés-Pineda y col., 2016). Los cambios en los patrones temporales de estos parámetros han sido identificados también en regiones de los Andes de Chile (Sarricolea y col., 2019).

La intensa erosión del suelo que se deriva del aumento de la intensidad de la lluvia es un problema crítico en muchas cuencas del mundo (Vrieling, Hoedjes y Velde, 2014; Mondal, Khare y Kun$\mathrm{du}, 2016)$. La cuenca de río Guayas (CRG), es la zona agrícola más fértil de Ecuador (Buckalew y col., 1998), y principal centro de producción de bienes agropecuarios. La distribución estacional y los totales anuales de precipitaciones son extremadamente irregulares, lo que ha provocado que la CRG se vea afectada por eventos de inundación y sequías, trayendo pérdidas económicas. Este es el caso de los años 1982 y 1983 que produjeron pérdidas estimadas de $3,18 \%$ y $28,63 \%$ del PIB, respectivamente (520 millones de dólares) (Egas, 1985). Mientras 
que en 1997-98 se perdieron $5664 \mathrm{~km}^{2}$ de producción agrícola (616,5 millones de dólares) (Corporación Andina de Fomento, 1998), causada por la marcada influencia del fenómeno de El Niño en la cuenca (Cadier y col., 1996); pero la zona de mayor afecta- ción es la cuenca baja, debido a que periódicamente se encuentra sometida a inundaciones que pueden tener consecuencias catastróficas, agravadas por acciones humanas como la deforestación y erosión en las cabeceras de los ríos (Rossel, Caddier y G., 1996).

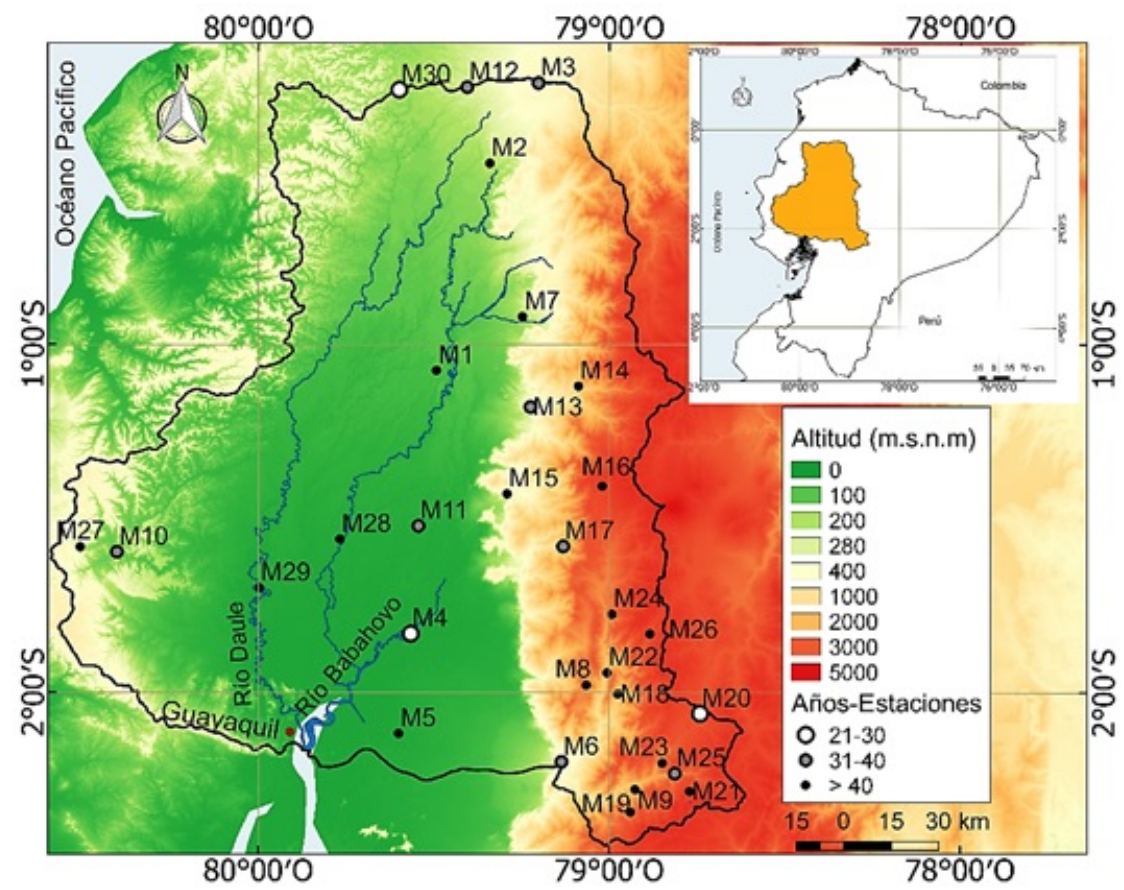

Figura 1. Localización de la cuenca del río Guayas, altitud, distribución de las 30 estaciones pluviométricas y años de estudio. El nombre de las estaciones se indica en la Tabla 1.

El río Guayas presenta problemas de sedimentación debido a la erosión de los suelos en la cuenca (Gobierno Provincial del Guayas, 2016), esto ha dado lugar a la formación de islotes en la unión del río Babahoyo y Daule (Figura 1), provocando erosión del cauce, deficiente drenaje e inundaciones frente a lluvias intensas, siendo este uno de los casos de inundaciones en la ciudad de Guayaquil (Figura 1) (Soledispa, 2002). Se ha recurrido a medidas como el dragado ante la acumulación de sedimentos que ascienden a $250 \mathrm{mil}$ toneladas por año (Gobierno Provincial del Guayas, 2018). No obstante, no se ha estudiado la lluvia como factor de erosividad en regiones ecuatoriales como la CRG. El objetivo de esta investigación es (a) regionalizar la precipitación para un período prolongado de datos de precipitación mensual (1968-2014) y (b) estimar la agresividad y concentración de las precipitaciones en la CRG.

\section{Materiales y métodos}

\section{1 Área de estudio}

La CRG se ubica en la parte central oeste del Ecuador $\left(0^{\circ} 14^{\prime}\right.$ a $2^{\circ} 27^{\prime} \mathrm{S}$ y $78^{\circ} 36^{\prime}$ a $\left.80^{\circ} 36^{\prime} \mathrm{O}\right)$ (Figura 1$)$. $\mathrm{El}$ área se caracteriza por una gradiente altitudinal significativa que va hasta los $4000 \mathrm{msnm}$, con una superficie de $32890 \mathrm{~km}^{2}$, correspondiente al $13 \%$ del Ecuador, y concentra $\approx 40 \%$ de la población del país (SENAGUA200). La CRG drena en el Golfo de Guayaquil, los principales ríos son el Daule y Babahoyo (Figura. 1) que se unen cerca de Guayaquil, la ciudad más grande de país Damanik-Ambarita2016. El río Guayas, el más grande de la costa occidental de Sudamérica, con un escurrimiento promedio anual de $1350 \mathrm{~m}^{3} / \mathrm{s}$ (Twilley y col., 2001), tiene un canal principal recto que se bifurca en una red de canales fluviales que recorren $30 \mathrm{~km}$ de manglares y plani- 
cies de marea (Reynaud y col., 2018). Las principales actividades económicas en la CRG son agricultura, pesca y generación de energía hidroeléctrica. Las principales presiones ambientales sobre el ecosistemas de agua dulce son la contaminación por aguas residuales, agricultura, cambios en el uso de la tierra y dos represas hidroeléctricas (Thi Nguyen y col., 2015). En los últimos años se han incrementado los problemas de sedimentación fluvial en la parte baja de la cuenca, considerado uno de los factores que contribuyen al riesgo de inundación ante lluvias extremas. Esta sedimentación se percibe localmente como una consecuencia de las intervenciones llevadas a cabo en la cuenca superior y eventos naturales como El Niño (Barrera-Crespo y col., 2018). Los impactos de El Niño en esta cuenca han provocado inundaciones (erosión por lluvia, deslizamiento y deslaves), contaminación del agua po-

\subsection{Metodología}

La metodología se compone de tres etapas resumidas en la Figura 2, el primero corresponde a la evaluación de los datos de precipitación, su homogenización y completación de datos mensuales por el método del vector regional (MVR); el segundo co- table, daños a la infraestructura y al sector agropecuario (Corporación Andina de Fomento, 1998).

\subsection{Datos}

Se recopilaron registros de precipitación de 250 estaciones meteorológicas provenientes del Instituto Nacional de Meteorología e Hidrología (INAMHI), cada una de ellas con diferentes períodos entre 1962 y 2016. Con el fin de asegurar la mayor disponibilidad de datos mensuales se seleccionaron 30 estaciones con un periodo de 47 años (1968-2014), el cual tuvo la menor cantidad $(<13 \%)$ de datos faltantes (Tabla 1). De las 30 estaciones, el $63 \%$ de ellas presenta más de 40 años de registros continuos, el $27 \%$ entre 31 y 40 años, mientras el $10 \%$ entre 21 y 30 años.

rresponde al proceso de regionalización por medio del agrupamiento de estaciones usando k-means y la interpolación usando co-kriging; y la última etapa corresponde a la determinación de la agresividad y concentración de las precipitaciones a través de diferentes índices.

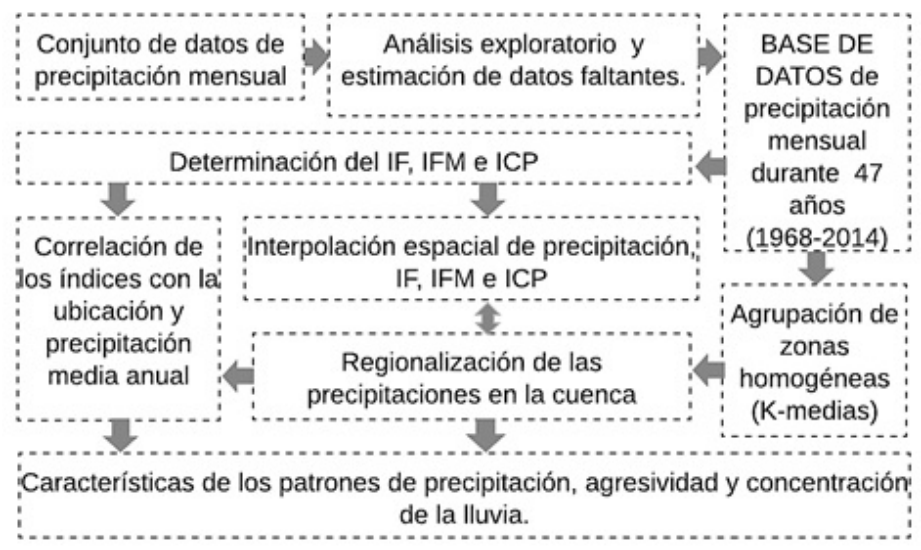

Figura 2. Esquema metodológico para la regionalización, agresividad y concentración de la serie de tiempo de lluvia.

\subsubsection{Método del vector regional}

Para evaluar la calidad y la estimación de datos faltante se utilizó el MVR, método que se orienta a la crítica, homogenización y completación-extensión de datos de precipitación (Hiez, 1977; Brunet-Moret,
1979). El MVR se basa en la creación de una estación 'Vector', tipo 'especie promedio'. Este concepto se refiere al cálculo de un promedio ponderado de anomalías de lluvia para cada estación, superando los efectos de estaciones con valores extremos y bajos de lluvia. Luego, se encuentran las técnicas de 
Tabla 1. Características de las 30 estaciones en la cuenca del río Guayas: nombre, ubicación geográfica y datos faltantes.

\begin{tabular}{|c|c|c|c|c|c|c|}
\hline Código & Nombre & $\begin{array}{l}\text { Latitud } \\
\left({ }^{\circ} \mathbf{S}\right)\end{array}$ & $\begin{array}{c}\text { Longitud } \\
\left({ }^{\circ} \mathrm{O}\right)\end{array}$ & $\begin{array}{l}\text { Altitud } \\
\text { (msnm) }\end{array}$ & $\begin{array}{c}\% \text { datos } \\
\text { faltantes }\end{array}$ & Período \\
\hline M1 & Pichilingue & $-1,07$ & $-79,49$ & 81 & 0 & $1968-2014$ \\
\hline M2 & Puerto Ila & $-0,48$ & $-79,34$ & 319 & 2 & $1968-2014$ \\
\hline M3 & Sto. Domingo Aeropuerto & $-0,25$ & $-79,20$ & 554 & 6 & 1968-1998 \\
\hline M4 & Isabel María & $-1,83$ & $-79,56$ & 4 & 8 & $1968-1988$ \\
\hline M5 & Milagro (Ingenio Valdez) & $-2,12$ & $-79,60$ & 23 & 0.2 & $1968-2014$ \\
\hline M6 & Bucay & $-2,20$ & $-79,13$ & 480 & 4 & $1969-2000$ \\
\hline M6 & San Juan La Mana & $-0,92$ & $-79,25$ & 215 & 9 & $1968-2014$ \\
\hline M8 & Chillanes & $-1,98$ & $-79,06$ & 2330 & 4 & $1968-2014$ \\
\hline M9 & Chunchi & $-2,28$ & $-78,92$ & 2177 & 3 & $1968-2014$ \\
\hline M10 & Camposano \#2 & $-1,59$ & $-80,40$ & 113 & 1 & $1977-2014$ \\
\hline M11 & Pueblo Viejo & $-1,52$ & $-79,54$ & 19 & 13 & $1976-2014$ \\
\hline M12 & Las Delicias-Pichincha & $-0,26$ & $-79,40$ & 340 & 8 & $1968-2002$ \\
\hline M13 & Moraspungo-Cotopaxi & $-1,18$ & $-79,22$ & 409 & 8 & 1968-87; 96-2014 \\
\hline M14 & Ramón Campaña & $-1,12$ & $-79,09$ & 1462 & 7 & $1968-2014$ \\
\hline M15 & Echeandia & $-1,43$ & $-79,29$ & 308 & 9 & $1968-2014$ \\
\hline M16 & Salinas-Bolívar & $-1,40$ & $-79,02$ & 3600 & 10 & $1969-2014$ \\
\hline M17 & Río San Antonio-Monjas & $-1,58$ & $-79,13$ & 2200 & 2 & $1980-2014$ \\
\hline M18 & Pallatanga & $-2,00$ & $-78,97$ & 1523 & 9 & $1968-2014$ \\
\hline M19 & Compud & $-2,34$ & $-78,94$ & 2402 & 8 & $1968-2014$ \\
\hline M20 & Palmira INAMHI & $-2,06$ & $-78,74$ & 3180 & 4 & $1968-1991$ \\
\hline M21 & Achupallas-Chimborazo & $-2,28$ & $-78,77$ & 3178 & 1 & $1968-2014$ \\
\hline M22 & Chimbo Pj Pangor & $-1,94$ & $-79,00$ & 1452 & 10 & $1968-2014$ \\
\hline M23 & Alausi & $-2,20$ & $-78,85$ & 2267 & 12 & $1968-2014$ \\
\hline M24 & Cañi-limbe & $-1,77$ & $-78,99$ & 2800 & 6 & $1969-2014$ \\
\hline M25 & Guasuntos & $-2,23$ & $-78,81$ & 2438 & 2 & $1975-2014$ \\
\hline M26 & Pangor-J.de Velasco & $-1,83$ & $-78,88$ & 3109 & 11 & $1970-2014$ \\
\hline M27 & Colimes de Pajan & $-1,58$ & $-80,51$ & 200 & 2 & $1970-2014$ \\
\hline M28 & Vinces INAMHI & $-1,56$ & $-79,77$ & 14 & 8 & $1968-2014$ \\
\hline M29 & La Capilla INAMHI & $-1,70$ & $-80,00$ & 7 & 5 & $1968-2014$ \\
\hline M30 & Palmeras Unidas (Palmar) & $-0,26$ & $-79,60$ & 460 & 10 & $1987-2012$ \\
\hline
\end{tabular}

interposición anual $Z_{i}$ y la precipitación anual de $P_{j}$, que se extienden por medio de la técnica de mínimos cuadrados. Esto podría obtenerse minimizando la suma de la siguiente ecuación (Espinoza Villar y col., 2009).

$$
\sum_{i=1}^{N} \sum_{j=1}^{M}\left(\frac{P_{i j}}{P_{j}}-Z_{i}\right)
$$

Donde $i$ es el índice de años, $j$ el índice de estaciones, $N$ el número de años y $M$ el número de estaciones. $P_{i j}$ representa la precipitación anual en la estación $j$, año $i ; P_{j}$ es el período de lluvia promedio extendido de $N$ años; y finalmente $Z_{i}$ es el índice pluviométrico regional del año $i$. El conjunto completo de valores $Z_{i}$ durante todo el período se co- noce como vector anual de índices pluviométricos regionales. Al ser un proceso iterativo, este método permite calcular el vector de cada una de las regiones predefinidas, luego proporciona una comparación de variabilidad interanual de estaciones - vector, para finalmente descartar aquellas que no son consistentes con el vector regional (VR). Este proceso se repite tanto como sea necesario y fue realizado empleando el software HYDRACCESS (Vauchel, 2005)

\subsubsection{Regionalización}

En este estudio se utilizó el método de k-means, ampliamente usado para regionalizar zonas homogéneas de precipitaciones (Golian y col., 2010; Gómez- 
Latorre, 2015; Shahana Shirin y Thomas, 2016; Rau y col., 2017). K-means es el algoritmo de agrupamiento más utilizado para identificar grupos homogéneos de objetos denominados conglomerados. Los datos dentro de un clúster comparten muchas características pero son muy diferentes a los datos que no pertenecen a ese clúster (Yashwant y Sananse, 2015). Los datos en este estudio se encuentran resumidos en una matriz de 30 filas correspondientes a las estaciones meteorológicas y 6 columnas con información como nombre de la estación, altitud, latitud, longitud y precipitación acumulativa. Una parte clave de la aplicación k-means es definir un número óptimo de grupos, que se puede realizar mediante la estimación del coeficiente de silueta $(S)$ para cada número de grupos; el coeficiente $S$ tiene la ventaja que sólo considera la partición real y no depende del algoritmo de agrupamiento, su valor se obtiene mediante la ecuación 2 (Rousseeuw, 1987):

$$
S_{(i)}=\frac{b_{i}-a_{i}}{\max \left[a_{(i)}, b_{(i)}\right]}
$$

Donde $a_{(i)}$ corresponde a la similitud promedio entre el objeto $i$ y los otros objetos del mismo grupo y $b_{(i)}$ es la similitud promedio entre el objeto i y los miembros de los k-clúster. El coeficiente de $S$ varía entre 1 y +1 ; la partición será mejor cuanto más cercano esté a +1 ; un valor negativo significa que no hay una buena correspondencia entre los miembros de su grupo, un valor de cero significa que el objeto podría pertenecer a cualquier grupo (Kaufman y Rousseeuw, 2005). También, se realizó la homogenización de la precipitación para eventos extremos como el Niño de 1997-1998, considerando la metodología anterior.

La interpolación de datos de precipitación anual se realizó utilizando un enfoque geoestadístico, método de co-kriging, el cual es una versión multivariable de la técnica de kriging (Goovaerts, 1998), considerando dos variables (altitud y precipitación acumulativa) transformadas logarítmicamente debido al sesgo y al amplio rango numérico de los valores de precipitación. Este método fue utilizado para la interpolación y la delimitación de zonas de precipitación (Rau y col., 2017) y para el mapeo de la distribución espacial de los índices.

\subsubsection{Análisis de agresividad climática y concentra- ción de las precipitaciones}

La agresividad climática se analizó mediante la interpretación del índice de Fournier (IF) e Índice de Fournier Modificado (IFM). Fournier, (1960) propone un índice de agresividad climática o IF, que presenta una alta correlación con la cantidad de sedimentos arrastrados por la escorrentía. El IF estima las características erosivas (agresividad) basadas en el mes más lluvioso de cada año dentro de un período de tiempo dado. Para el cálculo del IF se utilizó la expresión (3). Donde IF: Índice de Fournier para el año $j, p_{\text {máx }}$ es la precipitación media concerniente al mes más lluvioso $(\mathrm{mm})$ y $P$ es la precipitación media anual (mm).

$$
I F_{j}=\frac{p_{\text {máx }_{j}}^{2}}{P}
$$

Sin embargo, es necesario considerar zonas que presentan más de una máxima mensual o zonas donde los valores pluviométricos presentan valores elevados debido a la estacionalidad (Jordán y Bellinfante, 2000). Para corregir esos errores se propuso una modificación del IF original utilizando la precipitación acumulada, denominado IFM (Arnoldus, 1978). Este índice considera la lluvia de los doce meses y no sólo la del mes más lluvioso; su cálculo relaciona las precipitaciones mensuales con las anuales (ecuación 4). Donde $I F M_{j}$ es el índice de agresividad de la lluvia, para el año $j, p_{i j}$ es la precipitación mensual del mes $i(\mathrm{~mm})$ del año $j$ y $P_{m}$ es la precipitación media anual.

$$
I F M_{j}=\frac{\sum_{i=1}^{12}\left(p_{i j}\right)^{2}}{P_{m}}
$$

La estacionalidad de la precipitación se estimó por medio del Índice de Concentración de Precipitación (ICP) propuesto por Oliver, (1980), siendo un indicador de la distribución de la precipitación temporal y utilizado como un estimador del comportamiento extremo de la precipitación (Sarricolea, Araya y Salazar, 2014). Tradicionalmente se ha aplicado en una escala anual y describe si la precipitación anual se concentra temporalmente en un solo mes o si se distribuye uniformemente a lo largo del año. El ICP se calculó a una escala anual a partir de la ecuación 5.

$$
I C P_{j}=100 \frac{\sum_{i=j}^{12} p_{i j}^{2}}{P_{j}^{2}}
$$


El ICP fue analizado también a escala estacional considerando los períodos de mayor precipitación (diciembre-mayo) y menor precipitación (junio-noviembre), según la ecuación (6).

$$
I C P_{\mathrm{estac}}=1000 \frac{\sum_{i=j}^{6} p_{i j}^{2}}{\left(\sum_{i=j} P_{i j}\right)^{2}}
$$

Donde $I C P_{j}$ es el índice de concentración de las precipitaciones anuales (\%), para el año $j, I C P_{\text {estac }}$ es el índice de concentración a escala estacional $(\%)$, $p_{i j}$ es la precipitación del mes $i$ en el año $j, P_{j}$ es la precipitación anual del año $j$. La diferencia principal entre estos índices son los rangos de valores de clasificación mostrados en la Tabla 2.

Las clasificaciones de los índices son a partir de (Fournier, 1960; Arnoldus, 1978) y (Oliver, 1980), respectivamente. La influencia del cambio climático en el patrón estacional de la concentración de las precipitaciones fue determinada mediante la prueba estadística no paramétricas de Mann-Kendall (MK), a tres niveles de significación $(90 \%, 95 \%$ y $99 \%$ ). El análisis de MK se realizó empleando el software TREND (https://toolkit.ewater.org.au/ trend). La prueba de MK verifica la existencia de cambios positivos/negativos de una serie de datos, contra una hipótesis nula de no tendencias y donde los datos son aleatorios e independientes (Mann, 1945; Kendall, 1975). El análisis de tendencias de MK es una prueba robusta cuando los datos difieren de la 'normalidad' y menos sensibles a valores atípicos (Lanzante, 1996). El análisis de MK se ha utilizado ampliamente para el análisis de detección de tendencias meteorológicas e hidrológicas (Kumar y col., 2009; Gocic y Trajkovic, 2013; Hermida y col., 2015; Zeleňáková y col., 2016; Güçlü, 2018; Sarricolea y col., 2019).

\section{Resultados y discusiones}

\subsection{Regiones homogéneas}

El valor óptimo para los números de clúster se determinó mediante el valor promedio general de $\mathrm{S}$ y el número de $S$ negativo para cada grupo clúster que varía de 2 a 10 (Tabla 3). El valor máximo de $\mathrm{S}$ se obtuvo para el grupo de clúster $2(0,51)$ y con un número menor de silueta negativo (1), es el único grupo que se considera como una estructura razonable, porque su valor de $\mathrm{S}$ es mayor a 0,50 (Kononenko y Kukar, 2007). Internamente el grupo de clúster 2 presenta una estructura de agrupamiento fuerte $(\mathrm{S}=0.66)$ mientras que el clúster 1 alcanzó un valor menor, con un $\mathrm{S}$ negativo (Figura 3b). Esto indica que en el clúster 1 se pueden encontrar centros de agrupación, aunque existe un considerable 'ruido'. Los grupos de clúster para eventos extremos como el Niño de 97-98 presentó resultados similares (Tabla 3).

Tabla 2. Índices que determinan la agresividad y concentración de precipitaciones.

\begin{tabular}{|c|c|c|}
\hline Índice & & Clasificación \\
\hline \multirow{5}{*}{$\begin{array}{l}\text { Índice de Fournier } \\
\text { (IF) }\end{array}$} & $<50$ & Muy Bajo \\
\hline & $50-100$ & Bajo \\
\hline & $100-150$ & Moderado \\
\hline & $150-200$ & Alto \\
\hline & $>200$ & Muy alto \\
\hline \multirow{5}{*}{$\begin{array}{l}\text { Índice de Fournier } \\
\text { modificado (IFM) }\end{array}$} & $<100$ & Muy Bajo \\
\hline & $100-200$ & Bajo \\
\hline & $200-300$ & Moderado \\
\hline & $300-400$ & Alto \\
\hline & $>400$ & Muy alto \\
\hline \multirow{5}{*}{$\begin{array}{l}\text { Índice de concentración } \\
\text { de precipitación (ICP) }\end{array}$} & $8,3 \%-10 \%$ & Uniforme \\
\hline & $10 \% 15 \%$ & Moderadamente estacional \\
\hline & $15 \% 20 \%$ & Estacional \\
\hline & $20 \% \longleftarrow 50 \%$ & Fuertemente estacional \\
\hline & $50 \%-100 \%$ & Irregular \\
\hline
\end{tabular}


Las distribuciones espaciales de K-means (2) muestran un arreglo de estaciones de acuerdo con la variación topográfica y la longitud (Figura 3). El clúster de dos grupos divide a la CRG en dos regiones homogéneas de precipitación: parte baja y media (triángulos rojos) y parte alta (círculos negros). Las dos regiones están bien delineadas, teniendo en cuenta el mapa de interpolación de la lluvia, como se muestra en la Figura 3a.

La región uno (R1) se ubica en las laderas de la cordillera occidental de los Andes y las grandes llanuras de la costa ecuatoriana $\left(78,9^{\circ}\right.$ a $\left.80,59^{\circ} \mathrm{O}\right)$ (Figura 4a). La altitud varía entre 3 a $2500 \mathrm{msnm}$, ocupa el 85,2\% del área de la CRG. El régimen es unimodal, la estación lluviosa va de diciembre a mayo (Figura 4b) y concentra el $89 \%$ de la lluvia anual acumulada (Cadier y col., 1996; Rossel y Cadier, 2009; Fries y col., 2014) y una estación seca (junio - noviembre) (Hastenrath, 1997). El rango de precipitación va desde los 850 hasta los $3500 \mathrm{~mm}$ al año y un CV interanual de 0,38 (Figura 4a). La lluvia en esta región es de convección y la distribución de época seca y lluviosa se debe al movimiento de norte a sur de la Zona de Convergencia Intertropical (ZCIT) (Rollenbeck y Bendix, 2011).

En la región dos (R2) se sitúa en la cordillera occidental de los Andes; la altitud es superior a los $1500 \mathrm{msnm}$ e inferior a $4000 \mathrm{msnm}$. Los totales pluviométricos (450 a $1500 \mathrm{~mm}$ año-1) y el CV interanual (0.34) fueron relativamente bajos comparados con la R1 (Figura 4a). La distribución de la precipitación tiene una tendencia bimodal: el primer pico se presenta de enero a mayo, seguido de octubre a diciembre y el período mayo a agosto es el de menor precipitación media mensual (Figura 4c). La cantidad de lluvia que cae en esta zona se debe a la influencia de lluvia orográfica y de convección (Rollenbeck y Bendix, 2011). La formación de precipitaciones es compleja en las montañas debido a la interacción entre el transporte de humedad, el calentamiento de la superficie diferencial, el campo de viento sinóptico y el sistema local de brisa montañosa (Daly, Smith y Smith, 2007; Foresti y Pozdnoukhov, 2012).

Tabla 3. Resultados del análisis de k-medias para el número de grupos de clúster.

\begin{tabular}{cccccccccc}
\hline Grupos de Clúster & $\mathbf{2}$ & $\mathbf{3}$ & $\mathbf{4}$ & $\mathbf{5}$ & $\mathbf{6}$ & $\mathbf{7}$ & $\mathbf{8}$ & $\mathbf{9}$ & $\mathbf{1 0}$ \\
\hline Promedio general de silueta & 0,51 & 0,46 & 0,35 & 0,31 & 0,34 & 0,30 & 0,34 & 0,27 & 0,23 \\
\hline Número de silueta negativa & 1 & 3 & 3 & 3 & 2 & 3 & 3 & 4 & 6 \\
\hline Promedio general de silueta_eventos extremos & 0,52 & 0,40 & 0,26 & 0,29 & 0,29 & 0,26 & 0,26 & 0,26 & 0,26 \\
\hline Número de silueta negativa_eventos extremos & 0 & 3 & 4 & 5 & 6 & 5 & 4 & 5 & 5 \\
\hline
\end{tabular}




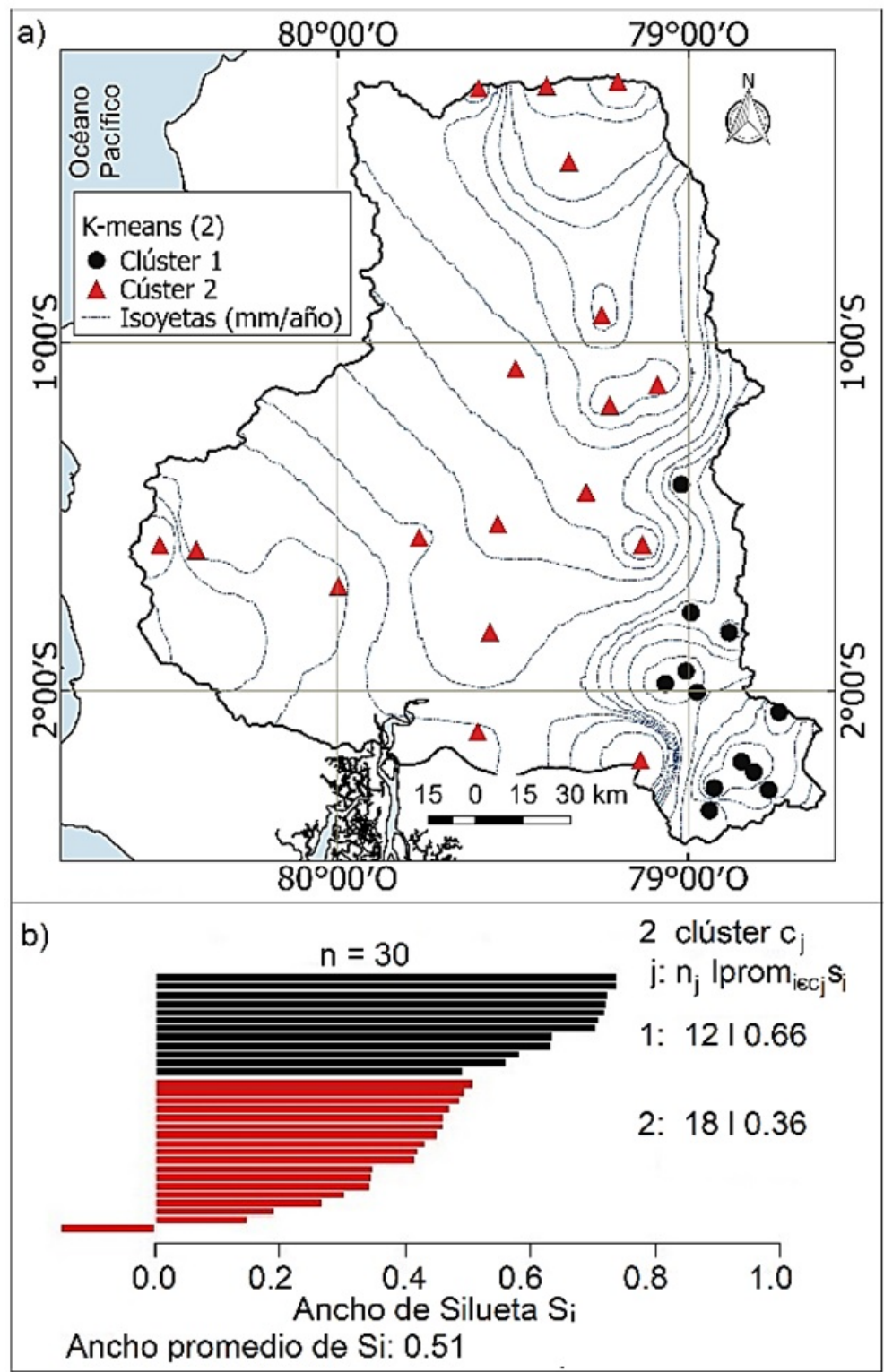

Figura 3. Distribución espacial del grupo de clúster (2) obtenido con el proceso k-means y su valor de silueta.

Se pueden identificar dos patrones de precipitación anual dentro del área de estudio: en la R1 se encontró un ciclo anual bien diferenciado entre períodos de avenidas y estiaje, caracterizado por picos para los años 72-73; 75-76; 91-92; y eventos extremos para 82-83 y 97-98 (Figura 4d). Las precipitaciones extremas en Ecuador son asociadas a eventos de El Niño que causaron severas inundaciones, pérdidas económicas y enfermedades (Bendix y Bendix, 2006). En la R1, la influencia del fenómeno de El Niño se encuentra fuertemente vinculada a los excedentes de lluvia anual (Rossel y col., 1998). La R2 presenta una débil estacionalidad, la cual es congruente con el coeficiente de variación promedio estimado $(0,34)$, donde se muestra una disminución de la precipitación promedio anual a lo largo de la mayoría de las estaciones en comparación a R1. Los eventos extremos (82-83 y 97-98) son también observados en la región R2, debido a que la influencia de El Niño en esta zona es variable (Cadier y col., 1996; Rossel y col., 1998). 


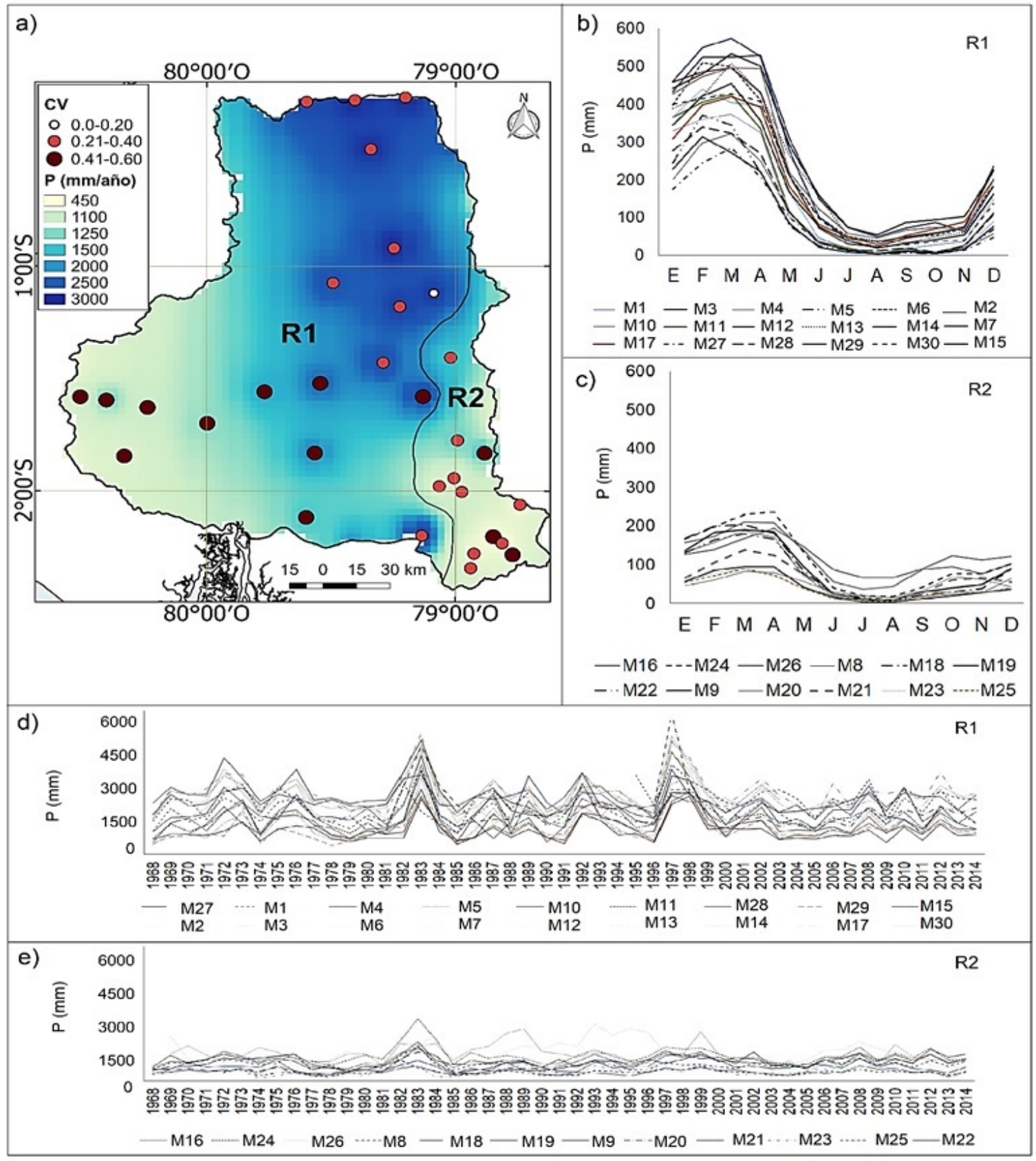

Figura 4. Distribución espacial de las dos regiones (R1-R2) de lluvia homogénea después del proceso de regionalización por k-means. a) Rango de coeficiente de variación (CV) interanual para las 30 estaciones pluviométricas. b) y c) Régimen de precipitación mensual de R1 y R2. d) y e) Distribución de precipitación anual (1968-2014) para las regiones R1 y R2.

\subsection{Agresividad Climática}

En la región R1, el $45 \%$ de las estaciones presentaron valores promedios anuales del IF superiores a 150, esto sugiere la ocurrencia de lluvia de muy alta erosividad (Figura 5a). El IF para la R2 muestra que el $50 \%$ de los valores promedios anuales son inferiores a 50 y los valores restantes fueron superiores a 50 e inferiores a 100, considerados como lluvia de muy baja y baja erosividad, respectivamente (Figura 5a). Los resultados a partir de IFM sugieren un patrón espacial similar al IF (Figura 5b). De hecho, 
en la región R1 son predominantes valores mayores a 300, asociado a una alta erosividad. Mientras la región R2 presenta valores aproximados a 100, lo que indica niveles bajos o muy bajos de erosividad. La diferencia de los índices estimados para las regiones R1 y R2 pueden estar asociados a la distribución espacial de la lluvia promedio anual (Figura 4a), lo que sugiere una alta influencia de la estacionalidad de las lluvias en ambas regiones (Figura $4 \mathrm{~d}$-e). Asimismo, la estacionalidad de la cuenca es corroborada con los resultados del ICP (Figura 5c), pues estos alcanzan valores alrededor de $20 \%$, lo que propone una clasificación predominantemente entre estacional y fuerte estacionalidad. Además, en la región R1 el IFM logra identificar ocho estaciones de muy alta erosividad, esto puede ser debido a que el IFM considera la lluvia de todos los doce meses y no sólo la del mes más lluvioso del año. Por lo que esta metodología podría ser más apropiada para caracterizar la severidad de las lluvia de la zona en estudio (Castelan-Vega y col., 2015).

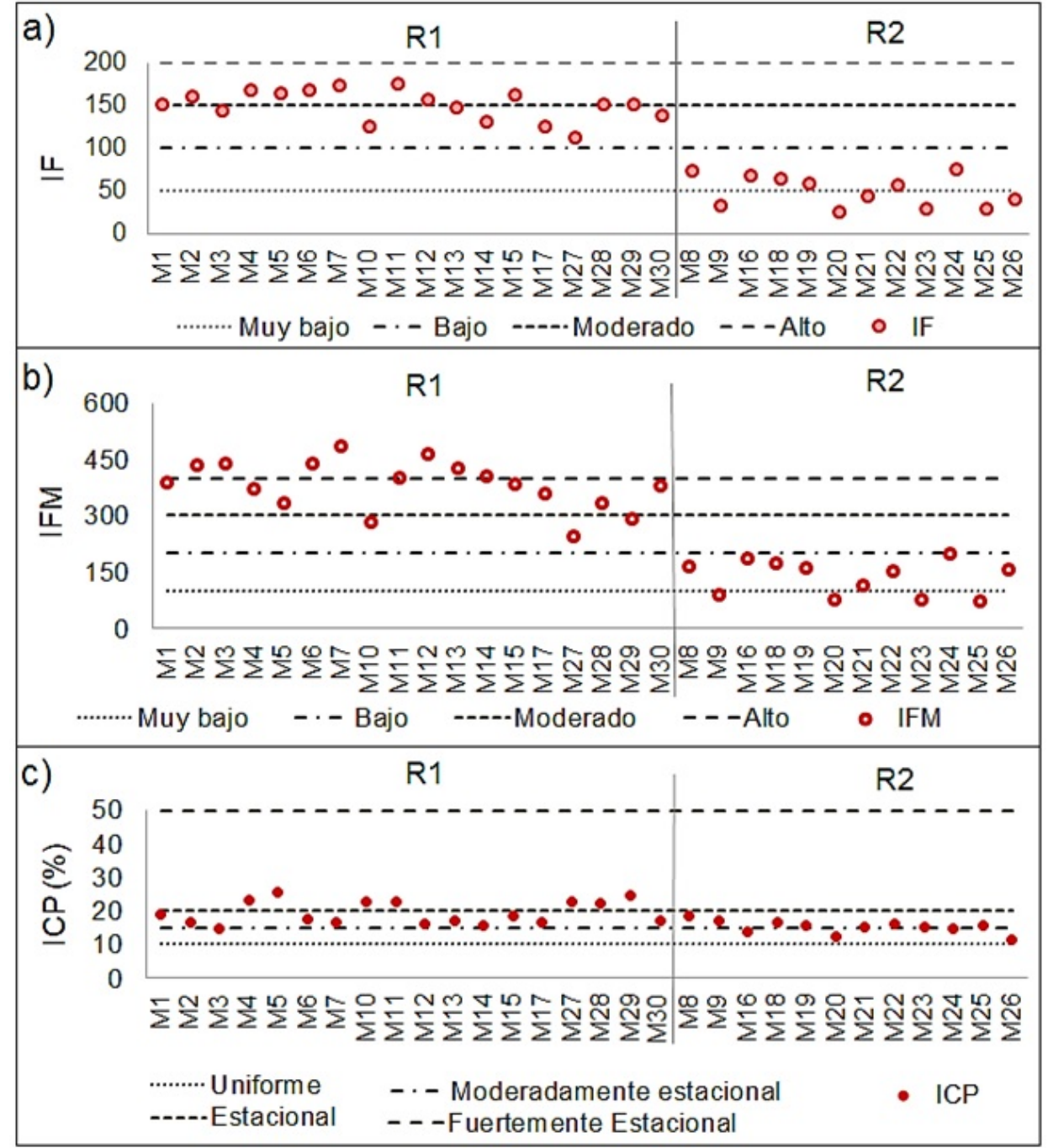

Figura 5. Índices de agresividad: a) índice de Fournier (IF), b) índice de Fournier Modificado (IFM) y c) concentración de la precipitación (ICP) media anual de 30 estaciones en el área de estudio. 
Es importante destacar que los resultados de agresividad en la región R2 coincide con la región de mayor precipitación anual, el cual presenta una buena correlación y nivel de significancia entre el patrón de precipitación media anual $(\mathrm{mm})$ con el IF $(r=0,77 ; p<0,01)$ e IFM $(r=0,93 ; p<0,01)$ (Figura $6 \mathrm{~d}$-e), lo que ratifica que una mayor acumulación anual $(\mathrm{mm})$ correspondería a una mayor agre- sividad (Besteiro y Delgado, 2011) en zonas donde la precipitación anual es mayor a $900 \mathrm{~mm}$ (Jordán y Bellinfante, 2000; Rey y col., 2012). También se observó una disminución del IF con la altitud $(r=0,85 ; p<0,01)$, no así para el IFM. Para la latitud y longitud no se encontró correlación entre los patrones espaciales de agresividad climática.

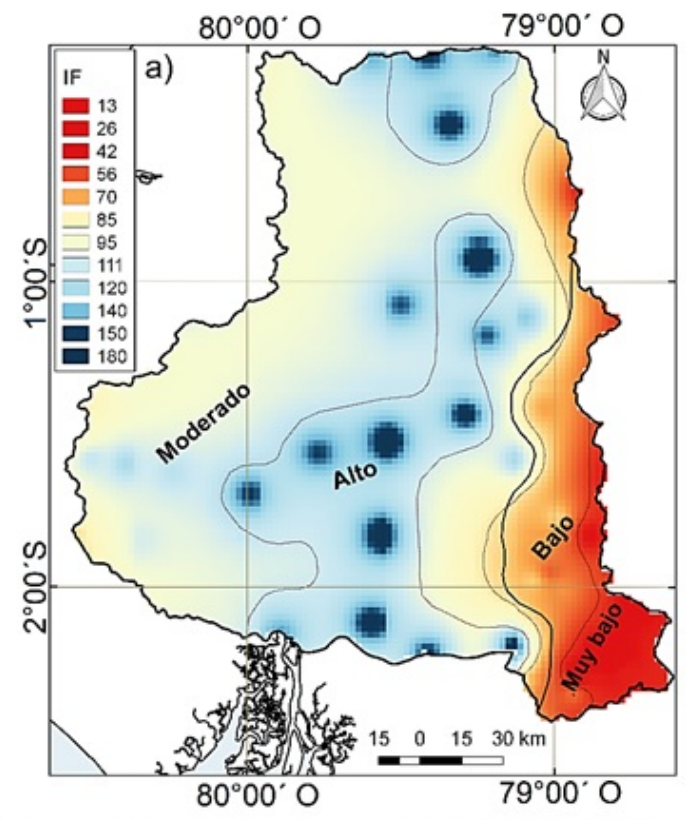

c) 200

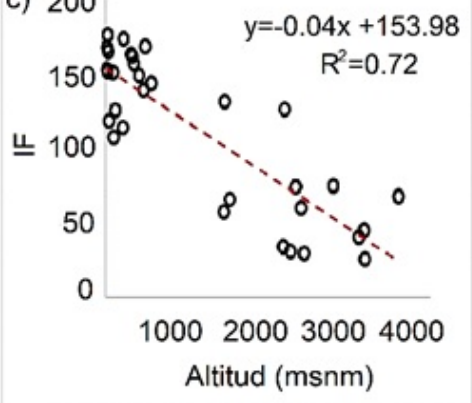

d) 200

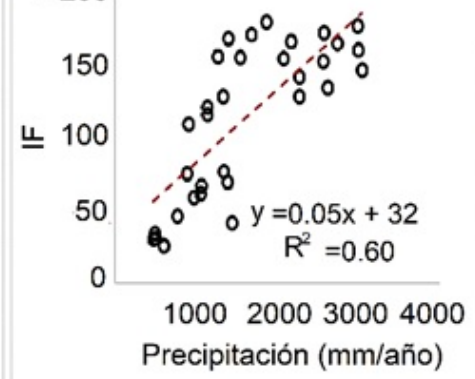

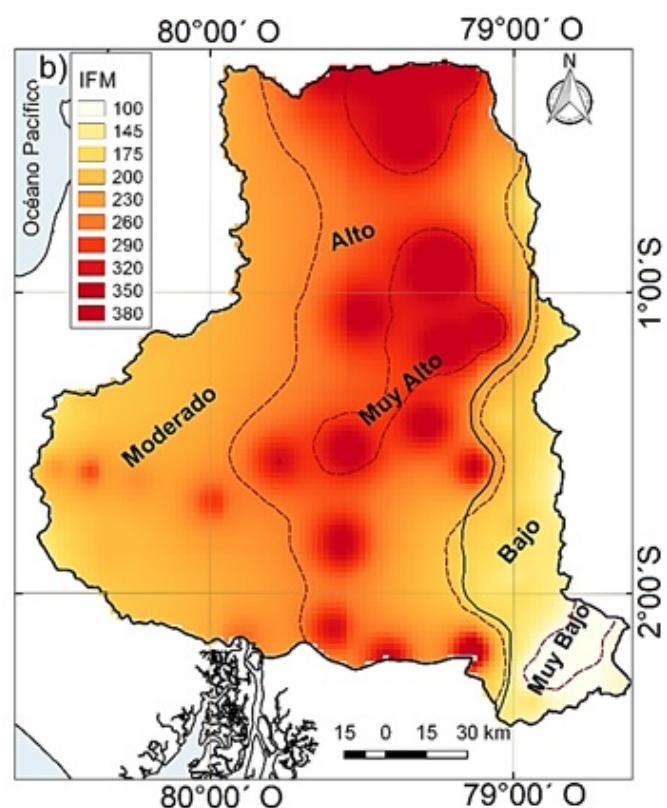

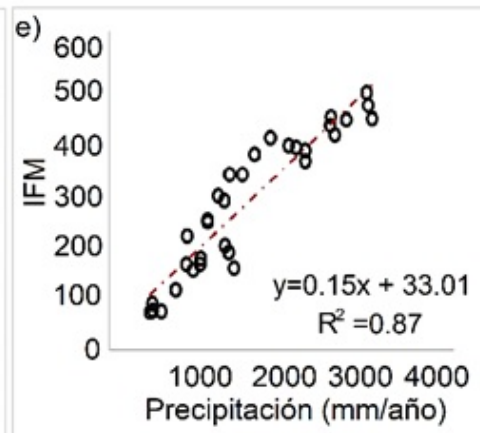

Figura 6. Distribución espacial: a) y b) Agresividad (IF, IFM) promedio anual para el período 1968-2014. Correlación: c) IF con la altitud, d) y e) IF e IFM con la precipitación acumulativa.

\subsection{Concentración de las precipitaciones}

La concentración media anual histórica observada en R1 se distribuyó de manera estacional con valores que oscilan entre $15-19 \%$, seguida de una distribución fuertemente estacional (Figura 5c); es decir, la precipitación se concentra en pocos meses del año. En la región R2, el ICP registra valores superiores a 11 e inferior a 18\%; 9 estaciones muestran una distribución estacional y 3 distribuciones moderadamente estacional a lo largo del año (Figura 5c). La concentración media anual histórica observada en regiones de alta montaña fue predominan- 
temente una concentración estacional y moderadamente estacional; estos resultados concuerdan con
Valdés-Pineda y col., (2016).

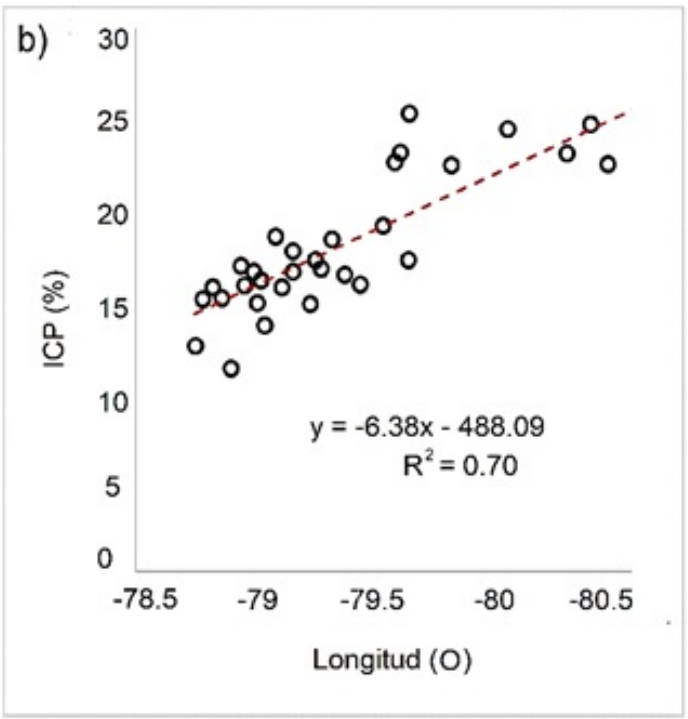

Figura 7. Distribución espacial: a) Concentración de las precipitaciones (ICP) promedio anual para el período 1968-2014 y b) Correlación del ICP con la longitud.

Las regiones de alta montaña presentan una concentración de lluvia entre estacional y moderadamente estacional, y la región central entre fuertemente estacional y estacional (Figura 7a). Esto sugiere una fuerte asociación con la longitud $(\mathrm{r}=0,83$; $\mathrm{p}<0,01$ ) (Figura $7 \mathrm{~b}$ ). La estacionalidad aumenta a lo largo de la gradiente longitudinal, lo que conlleva a una concentración más uniforme de precipitación anual. Los cambios en el ICP son complejos, posiblemente relacionados con las características atmosféricas globales y con factores locales y sinópticos que afectan la precipitación. Sin embargo, no se encontró correlación entre ICP con la latitud y la precipitación media anual, lo que sugiere que los años con mayor precipitación anual no están relacionados con la concentración de precipitación. Los hallazgos de esta investigación explican el porqué la R2 puede ser fuertemente afectada por la lluvia estacional durante el período de avenidas, donde la precipitación que se concentra en un número reducido de meses es relevante para la ocurrencia de erosión de suelos en la parte alta de la cuenca, ocasionando sedimentación en el área urbana ubicada en la parte baja de la CRG.
Para analizar los cambios en la concentración estacional de lluvia mensual, la serie de ICP estacional (período de mayor y menor precipitación) entre 1968 y 2014 fue estimada para las regiones R1 y R2 (Figura 8). Porcentajes de valores de concentración alrededor de $50 \%$ sugieren irregularidad de las precipitaciones; es decir, una alta cantidad de lluvia puede precipitar en un número reducido de meses, el cual es asociado a eventos de inundación. Por el contario, una muy baja cantidad de precipitación puede estar cayendo en un mayor número de meses, causando períodos de sequía que pueden afectar cultivos de secano. Esta irregularidad de la lluvia mensual es detectada en algunos años en el período seco de la región R1 (Figura 8). Esto podría afectar la agricultura por secano en la región oeste de la cuenca $(\approx 1600 \mathrm{msnm})$. Los resultados de ICP estacional $(\approx 20 \%)$ en el período de lluvias en $\mathrm{R} 1 \mathrm{y}$ R2 no muestran cambios temporales significativos y sugieren una estacionalidad marcada.

Los resultados del análisis de tendencias de MK permitió identificar las tendencias anuales positivas 
del $I C P_{\text {estac }}$ solo para el período de estiaje (junionoviembre) y tendencias negativas para los períodos de estiaje y de lluvias. Sin embargo, la mayoría de las estaciones no muestran una tendencia signifi- cativa (Figura 9 a y b). Las tendencias negativas del $I C P_{\text {estac }}$ se concentran para la R1 y R2 en las parte norte y sureste de la CRG.

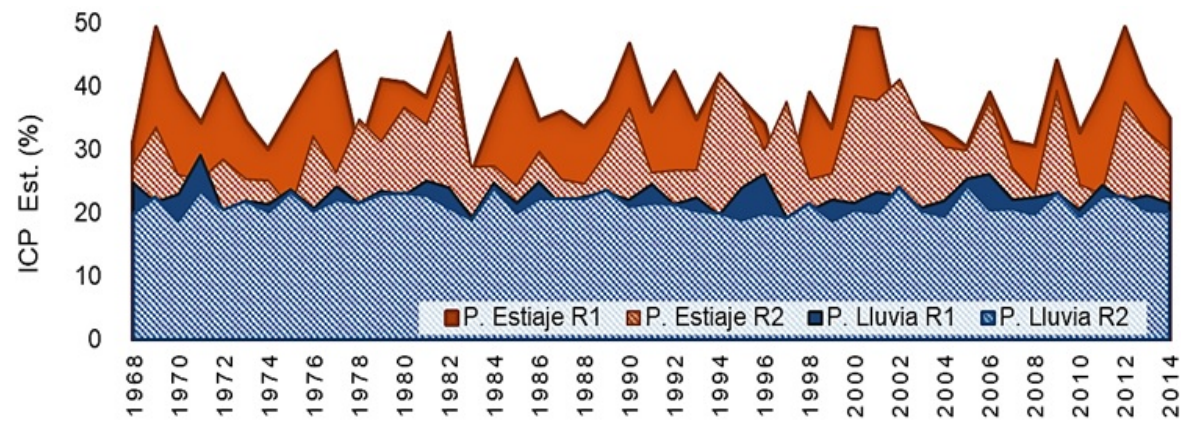

Figura 8. Serie anual de índice de concentración de la precipitación estacional $\left(I C P_{\text {estac }}\right)$ para el período $(P$.) de estiaje (junio a noviembre) y de lluvia (diciembre-mayo), 1968-2014 para la R1 y R2.

Para el período de fuertes precipitaciones (diciembre-mayo), solo dos estaciones muestran tendencia negativa en R1 y R2, con niveles de significancia del $90 \%$ y $99 \%$, respectivamente (Figura 8a). En el período de estiaje, dos estaciones muestran tendencia significativa negativa al 90 y $95 \%$ en la región sur de R1. Asimismo, solo una estación ubicada en la región de alta montaña registra tendencia positiva (Figura 8b). En general, las tendencias positivas o negativas identificadas en R1 y R2 indican cambios asociados a la irregularidad de la lluvia mensual en la distribución temporal de la concentración; no obstante, esta irregularidad es identificada en muy pocas estaciones.

\section{Conclusiones}

El estudio de la agresividad de las precipitaciones para la cuenca del río Guayas-Ecuador permitió homogenizar las zonas de precipitación, caracterizar la variabilidad de las lluvias en el período 1968 2014 y su potencial impacto erosivo. Los resultados sugieren dos regiones 1) al oeste de la cuenca en la región costanera hasta los $2500 \mathrm{msnm}$ y 2) al este de la cuenca en la región de alta montaña entre 1500 y $4000 \mathrm{msnm}$. La cuenca se evaluó de forma anual mediante el índice de Fournier (IF), índice de Fournier Modificado (IFM) y el Índice de concentración de precipitación (ICP). Los hallazgos de esta investigación a partir de IF e IFM indican que la cuenca del río Guayas en la zona costanera se clasifica como región de agresividad alta a muy alta en la región costera, mientras la región de alta montaña es clasificada como de baja o muy baja erosividad.

Las zonas de alta actividad agrícola ubicadas en la región costera presentan mayor potencial erosivo de la lluvia en comparación a la región de alta montaña. La distribución espacial de la concentración de las precipitaciones se incrementa de este a oeste, mostrando niveles de moderada a fuerte estacionalidad. Los resultados mediante los índices de agresividad y concentración de las precipitaciones permitieron evaluar cualitativamente el posible impacto de la lluvia sobre el suelo e identificar patrones de agresividad con la acumulación de la precipitación y concentración de la precipitación asociada a la longitud, lo que puede estar asociado a incursiones de flujos de humedad estacional proveniente de la Amazonía ecuatorial a los Andes (Espinoza Villar y col., 2009). Estos resultados también indican que la concentración de lluvia mensual no presenta cambios o tendencias predominantes entre 1968 y 2014. No obstante, se recomienda el análisis de la concentración de lluvia diaria en regiones ecuatoriales, ya que grandes porcentajes de lluvia estacional o anual pueden precipitar en pocos días. 

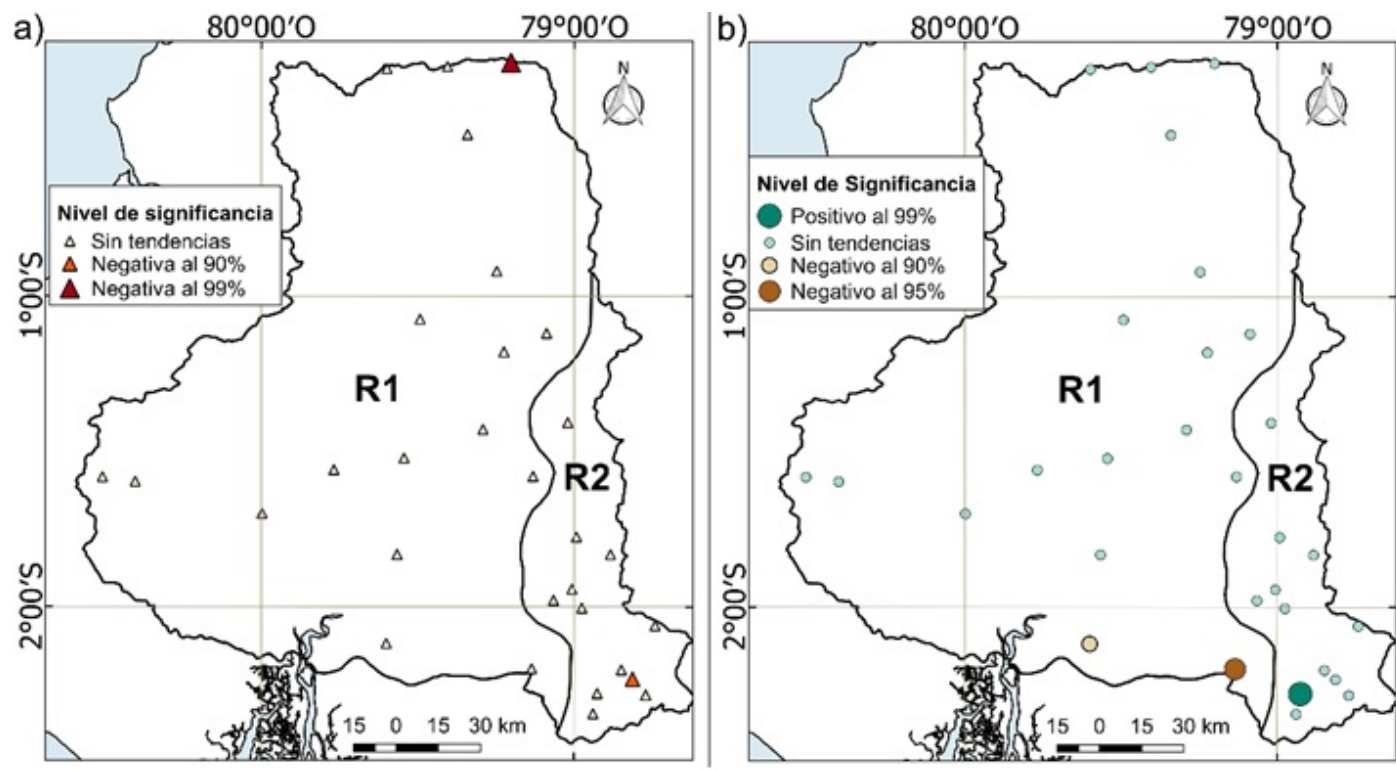

Figura 9. Distribución estacional de tendencias del ICP (1968-2014) en la cuenca del río Guayas para diferentes rangos del nivel de significancia: a) diciembre - mayo, b) junio - noviembre.

\section{Referencias}

Angulo-Martínez, M. y col. (2009). «Mapping rainfall erosivity at a regional scale, a comparison of interpolation methods inthe Ebro Basin (NE Spain)». En: Hydrol. Earth Syst. Sci. 13.10, 1907-1920. Online: https://bit.ly/33ucq9c.

Arnoldus, H. (1978). «Assessment of erosion». En: ed. por M. De Boodst y D. Gabriels. Chichester, England: John Wiley y Sons, Inc. Cap. An approximation of the rainfall factor in the Universal Soil Loss Equation.

Ballari, D. y col. (2018). «Spatial functional data analysis for regionalizing precipitation seasonality and intensity in a sparsely monitored region: Unveiling the spatio-temporal dependencies of precipitation in Ecuador». En: International Journal of Climatology 38, 3337-3354. Online: https: //doi.org/10.1002/joc.5504.

Barrera-Crespo, P.D. y col. (2018). «Sediment budget analysis of the Guayas River using a processbasedmodel.» En: Hydrol. Earth Syst. Sci. Discuss, 1-21. Online: https: / / doi.org / 10.5194 / hess \bibrangedash 2018\bibrangedash 467 .

Bendix, A. y J. Bendix (2006). «Heavy rainfall episodes in Ecuador during El Niño events and associated regional atmospheric circulation and SST patterns». En: Adv. Geosci. 6, 43-49. Online: https: //bit.ly/2H3ejiB.

Besteiro, S. y M. Delgado (2011). «Evaluación de la agresividad de las precipitaciones en la cuenca del Arroyo El Pescado, provincia de Buenos Aires (Argentina)». En: Revista de la Facultad de Agronomía, La Plata 110, 82-90. Online: https: / / bit.ly / 2MJ7Fnj.

Brunet-Moret, Y. (1979). «Homogénéisation des precipitation». En: Cahiers ORSTOM. Serie Hydrologie. 16.3-4, 147-170. Online: https: / / bit.ly / 2Lfg7Yg.

Buckalew, J.O. y col. (1998). Water Resources Assessment of Ecuador. Inf. téc. US Army Corps of Engineers (USACE).

Cadier, E. y col. (1996). Las inundaciones en la zona costera ecuatoriana: mecanismos responsables, obras de proteccion existentes y previstas. Inf. téc. INESEQ 28.

Castelan-Vega, R. y col. (2015). «Agresividad de las precipitaciones en la subcuenca del río San Marcos, Puebla, México.» En: Investigaciones Geográficas 83, Online: https: / / doi.org/10.14350/rig. 33480 .

Corporación Andina de Fomento (1998). «(1998) El Fenómeno El Niño 1997-1998.» En: Memorias, Retos y Soluciones. Vol. IV. Ecuador, págs. 72-73. 
Daly, C., J.W. Smith y McKane R.B Smith J.I. (2007). «High-Resolution Spatial Modeling of Daily Weather Elements for a Catchment in the Oregon Cascade Mountains, United States». En: Journal of Applied Meteorology and Climatology 46, 1565-1586. Online: https: / / doi.org / 10.1175 / JAM2548.1.

De Luis, M. y col. (2011). «Precipitation concentration changes in Spain 1946-2005». En: Natural Hazards and Earth System Science 11, 1259-1265. Online: https : / / doi . org / 10 . 5194 / nhess \bibrangedash $11 \backslash$ bibrangedash $1259 \backslash$ bibrangedash 2011.

Egas, R. (1985). «Ecuador, inundaciones 1982 - 1983 en la Cuenca baja del Guayas: Procesos de organizacion de los campesinos para hacer frente al desastre». En: Desastres Naturales y Sociedad en América Latina. Ed. por et al. J. Hardoy. Grupo Editora Latinoamericana.

Espinoza Villar, J.C. y col. (2009). «Spatio-temporal rainfall variability in the Amazon basin countries (Brazil, Peru, Bolivia, Colombia, and Ecuador)». En: International Journal of Climatology 29.11, 1574-1594. Online: https: / / doi.org / 10. 1002/joc.1791.

Ezenwaji, E., C. Nzoiwu y G. Chima (2017). «Analysis of Precipitation Concentration Index (PCI) for Awka Urban Area, Nigeria». En: Hydrology: Current Research 8.4, 287. Online: https: / / doi . org/10.4172/2157\bibrangedash 7587.1000287.

Foresti, L. y A. Pozdnoukhov (2012). «Exploration of alpine orographic precipitation patterns with radar image processing and clustering techniques: Exploration of alpine orographic precipitation patterns». En: Meteorological Applications 19, 407-419. Online: https://doi.org/10.1002/met. 272.

Fournier, F. (1960). Climat et erosion; la relation entre l'erosion du sol par l'eau et les precipitations atmospheriques. 1 ed. Paris, France. Online: https:/ / bit. ly/2L0sM2j: Presses universitaires de France.

Fries, A. y col. (2014). «Meteorology and Atmospheric Physics». En: Catchment precipitation processes in the San Francisco valley in southern Ecuador: combined approach using high-resolution radar images and in situ observations 126.1-2, 13-29. Online: https:/ / doi.org/10.1007/s00703 \bibrangedash $014 \backslash$ bibrangedash $0335 \backslash$ bibrangedash 3.

García-Barrón, L., J. Morales y A. Sousa (2018). «A new methodology for estimating rainfall aggressiveness risk based on daily rainfall records for multi-decennial periods». En: Science of The Total Environment 615.15, 564-571. Online: https : //doi.org/10.1016/j.scitotenv.2017.09.305.

Gobierno Provincial del Guayas (2016). Plan Provincial de Riego y Drenaje del Guayas. Gaceta oficial del Gobierno Autónomo Descentralizado Provincial del Guayas. Año 1. Inf. téc. 90. Guayaquil.

- (2018). Estudio de impacto ambiental del proyecto dragado de la II fase y disposición de los sedimentos de los alrededores del islote el palmar en la provincia del Guayas considerando como sitios de depósito Isabel Ana, lotización El Tejar, Caracoles y Terrenos particulares del cantón Durán. Inf. téc.

Gocic, M. y S. Trajkovic (2013). «Analysis of changes in meteorological variables using Mann-Kendall and Sen's slope estimator statistical tests in Serbia». En: Global and Planetary Change 100, 172-182. Online: https: / / doi .org / 10.1016/j. gloplacha.2012.10.014.

Golian, S. y col. (2010). «Comparison of classification and clustering methods in spatial rainfall pattern recognition at Northern Iran». En: Theoretical and Applied Climatology 102, 319-329. Online: https : / / doi . org / 10 . 1007 / s00704 \bibrangedash 010 \bibrangedash 0267\ bibrangedash $\mathrm{x}$.

Goovaerts, P. (1998). «Geostatistics for Natural Resources Evaluation». En: Geological Magazine 135.6, 819-842. Online: https:/ / bit.ly/30Jsxho.

Gregori, E., M.C. Andrenelli y G. Zorn (2006). «Assessment and classification of climatic aggressiveness with regard to slope instability phenomena connected to hydrological and morphological processes». En: Journal of Hydrology 329, 489-499. Online: https:/ /doi.org/10.1016/j.jhydrol.2006. 03.001.

Güçlü, Y.S. (2018). «Multiple Şen-innovative trend analyses and partial Mann-Kendall test». En: Journal of Hydrology 566, 685-704. Online: https: //doi.org/10.1016/j.jhydrol.2018.09.034.

Gómez-Latorre, Douglas (2015). «Regionalización de patrones de precipitación para periodos multianuales secos y humedos en el Altiplano Cundiboyacense». En: Online: https : / / bit . ly / 327Z9BO.

Hastenrath, S. (1997). «Annual cycle of upper air circulation and convective activity over the tropical Americas». En: Journal of Geophysical Research: Atmospheres 102, 4267-4274. Online: https:/ / doi. org/10.1029/96JD03122. 
Hermida, L. y col. (2015). "Hailfall in southwest France: Relationship with precipitation, trends and wavelet analysis». En: Atmospheric Research 156, 174-188. Online:https:/ / doi.org/10.1016/j. atmosres.2015.01.005.

Hernando, D. y M.G. Romana (2016). «Estimate of the (R) USLE rainfall erosivity factor from monthly precipitation data in mainland Spain». En: J. Iber. Geol. 42, 113-124. Online: https:/ / doi. org/10.5209/rev_JIGE. 2016.v42. n1.49120.

Hiez, G. (1977). «Lhomogénéité des données pluviométriques». En: Cahiers ORSTOM. Serie Hydrologie 14.2, págs. 111-119.

Jordán, A. y N. Bellinfante (2000). «Cartografía de la erosividad de la lluvia estimada a partir de datos pluviométricos mensuales en el Campo de Gibraltar (Cádiz)». En: Edafología 7.3, 83-92.

Kaufman, L. y P.J. Rousseeuw (2005). Finding groups in data: an introduction to cluster analysis. Wiley series in probability and mathematical statistics. Hoboken, N.J.: Wiley.

Kendall, M.G. (1975). Rank Correlation Methods. London, UK: Griffin.

Kinnell, P. (2010). «Event soil loss, runoff and the Universal Soil Loss Equation family of models: review». En: J. Hydrol. 385.

Kononenko, I. y M Kukar (2007). Machine Learning and Data Mining: Introduction to Principles and Algorithms. Chichester, UK. Online: https:/ / bit.ly / 2L0pL1Q: Horwood Publishing.

Kumar, S. y col. (2009). "Streamflow trends in Indiana: Effects of long term persistence, precipitation and subsurface drains». En: Journal of Hydrology 374, 171-183. Online:https://doi.org/10.1016/j.jhydrol.2009.06.012.

Lanzante, J.R. (1996). «Resistant, robust and nonparametric techniques for the analysis of climate data: Theory and examples, including applications to historical radiosonde station data». En: International Journal of Climatology 16.11, 1197-1226. Online: https:/ / bit.ly/2OQzgok.

Mann, H.B. (1945). «Nonparametric tests against trend». En: Econometrica 13, 245-259. Online: https://bit.ly/2ZvPQOj.

Martín-Fernández, L. y M. Martínez-Núñez (2011). "An empirical approach to estimate soil erosion risk in Spain». En: Science of The Total Environment 409, 3114-3123. Online: https: / / doi.org / 10.1016/j.scitotenv.2011.05.010.

Mondal, A., D. Khare y S. Kundu (2016). «Change in rainfall erosivity in the past and future due to climate change in the central part of India». En: International Soil and Water Conservation Research 4.3, 186-194. Online: https: / / doi:10.1016 / j. iswcr.2016.08.004.

Oliver, J. (1980). «Monthly precipitation distribution: a comparative index». En: The Professional Geographer 32.3, 300-309. Online: https: / / doi: $10.1111 / \mathrm{j} .0033 \backslash$ bibrangedash 0124.1980. 00300. $\mathrm{x}$.

Panagos, P. y col. (2015). "Rainfall erosivity in Europe». En: Science of The Total Environment 511, 801 -814. Online: https: / / doi.org / 10.1016/j. scitotenv.2015.01.008.

Parracho, A.C., P. Melo-Gonçalves y A. Rocha (2016). «Regionalisation of precipitation for the Iberian Peninsula and climate change». En: Physics and Chemistry of the Earth, Parts A/B/C 94, 146-154. Online: https: / / doi.org / 10.1016/j. pce.2015.07.004.

Prakash, S. y col. (2015). «Error characterization of TRMM Multisatellite Precipitation Analysis (TMPA-3B42) products over India for different seasons». En: Journal of Hydrology 529, 1302-1312. Online: https: / / doi.org / 10.1016/ j.jhydrol.2015.08.062.

Rasel, A.H., M. Islam y M. Keramat (2016). «Analysis of Annual and Seasonal Precipitation Concentration Index of North-Western Region of Bangladesh». En: International Conference on Computer, Communication, Chemical, Materials and Electronic Engineering, 107-110. Online:https: / / bit.ly/349Ewa3.

Rau, P. y col. (2017). «Regionalization of rainfall over the Peruvian Pacific slope and coast: Regionalization of Rainfall (Peruvian Coast)». En: International Journal of Climatology 37, 143-158. Online: https:/ /doi.org/10.1002/joc.4693.

Rey, J.C. y col. (2012). «Análisis de la agresividad y concentración de las precipitaciones en Venezuela. IV. región los Andes». En: BioAgro 24, 115 -120. Online:https://bit.ly/2MIEaSv.

Reynaud, J.-Y. y col. (2018). «Tide-dominated deltas in active margin basins: Insights from the Guayas estuary, Gulf of Guayaquil, Ecuador». En: Marine Geology 403, 165-178. Online: https: / / doi.org/10.1016/j.margeo.2018.06.002.

Rollenbeck, R. y J. Bendix (2011). «Rainfall distribution in the Andes of southern Ecuador derived from blending weather radar data and meteorological field observations». En: Atmospheric Re- 
search 99, 277-289. Online: https:/ / doi.org/10. 1016/j.atmosres.2010.10.018.

Rossel, F., E. Caddier y Gómez G. (1996). «Las inundaciones en la zona costera ecuatoriana: causas; obras de protección existentes y previstas». En: Bull. Inst. fr. études andines 25.3, 399-420.

Rossel, F. y E. Cadier (2009). «El Niño and prediction of anomalous monthly rainfalls in Ecuador». En: Hydrological Processes 23, 3253-3260. Online: https:/ / doi.org/10.1002/hyp.7401.

Rossel, F. y col. (1998). «Régionalisation de l'influence du El Nino sur les précipitations de l'Equateur». En: Bull. Inst. fr. études andines 27.3, 643-654. Online: https:/ / bit.ly/2zoRFOD.

Rousseeuw, P.J. (1987). «Silhouettes: A graphical aid to the interpretation and validation of cluster analysis». En: Journal of Computational and Applied Mathematics 20, 53-65. Online: Online:https: / / doi . org / 10 . 1016 / $0377 \backslash$ bibrangedash 0427(87)90125 \bibrangedash 7.

Sanchez-Moreno, J.F., C.M. Mannaerts y V. Jetten (2014). "Rainfall erosivity mapping for Santiago Island, Cape Verde». En: Geoderma 217, 74-82. Online: https: / / doi.org / 10.1016/j.geoderma. 2013.10.026.

Sarricolea, P. y col. (2019). «Trends of daily precipitation concentration in Central-Southern Chile». En: Atmospheric Research 215, 85-98. Online: https: / / doi.org / 10.1016/j.atmosres.2018.09. 005.

Sarricolea, Pablo, Cristobal Araya y Pamela Salazar (2014). «Concentración de las precipitaciones en Chile central y su relación con los patrones de variabilidad de baja frecuencia, período 1965-2010». En: 305-314. Online:https:/ / bit.ly / 30J8DmS.

Sevruk, B. (2004). Precipitation as the water cycle element. Theory and Practice of Precipitation Measurement. Inf. téc. (in German), Manuscript to be published.

Shahana Shirin, A.H. y R. Thomas (2016). «Regionalization of Rainfall in Kerala State». En: Procedia Technology 24, 15-22. Online:https:/ / doi.org/10. 1016/j.protcy.2016.05.004.

Soledispa, B. (2002). «Estudio de los sedimentos del sector donde convergen los ríos Daule y Babahoyo, y las posibles causan que están formando un nuevo islote en ese sector». En: Acta oceanográfica del Pacífico 11.1, págs. 185-201.

Terranova, O. y col. (2009). «Soil erosion risk scenarios in the Mediterranean environment using
RUSLE and GIS: an application model for Calabria (southern Italy)». En: Geomorphology 112, 228-245. Online: https: / / doi.org / 10.1016/j. geomorph.2009.06.009.

Thi Nguyen, T.H. y col. (2015). «Habitat suitability of the invasive water hyacinth and its relation to water quality and macroinvertebrate diversity in a tropical reservoir». En: Limnologica 52, 67-74. Online:https:/ / doi.org/10.1016/j.limno. 2015.03.006.

Twilley, R. R. y col. (2001). "The Gulf of Guayaquil and the Guayas River Estuary, Ecuador». En: Coastal Marine Ecosystems of Latin America. Ed. por Ulrich Seeliger y Björn Kjerfve. Berlin, Heidelberg: Springer Berlin Heidelberg, 245-263. Online: https : / / doi . org / 10 . 1007 / 978 \bibrangedash $3 \backslash$ bibrangedash 662 \ bibrangedash 04482 \bibrangedash 7_18.

Valdés-Pineda, R. y col. (2016). «Spatio-temporal trends of precipitation, its aggressiveness and concentration, along the Pacific coast of South America (36-49 $\mathrm{S}) »$. En: Hydrological Sciences Journal 61, 2110-2132. Online:https: / / doi.org / 10.1080/02626667.2015.1085989.

Vauchel, P. (2005). Hydraccess: Software for Management and processing of Hydro-meteorological data software, Version 2.1.4.

Vrieling, A., J. C. B. Hoedjes y M. van der Velde (2014). "Towards large-scale monitoring of soil erosion in Africa: Accounting for the dynamics of rainfall erosivity». En: Global and Planetary Change 115, 33-43. Online:https//doi:10.1016/j. gloplacha.2014.01.009.

Yashwant, S. y S.L. Sananse (2015). «Comparisons of Different Methods of Cluster Analysis with Application to Rainfall Data». En: International Journal of Innovative Research in Science, Engineering and Technology 4.11, 10861-10872. online: https: / / bit.ly/347RXre.

Zeleňáková, M. y col. (2016). «Monthly Trends of Precipitation in Gauging Stations in Slovakia». En: Procedia Engineering 162, 106-111. Online: https://doi.org/10.1016/j.proeng.2016.11.023.

Zubieta, R. y col. (2015). «Impacts of Satellite-based Precipitation Datasets on Rainfall-Runoff Modeling of the Western Amazon Basin of Peru and Ecuador». En: Journal of Hydrology 528, 599-612. Online: https:/ / bit.ly/30J71JQ.

Zubieta, R. y col. (2016). «Spatial analysis and temporal trends of daily precipitation concentration in the Mantaro River basin: central Andes of 
Peru». En: Stochastic Environmental Research and Risk Assessment, 1-14. Online: https / / doi:10. 1007 / s00477 \bibrangedash 016 \bibrangedash $1235 \backslash$ bibrangedash 5.
Zubieta, R. y col. (2019). «Assessing precipitation concentra-tion in the Amazon basin from different satellite-based datasets». En: Int. J. Climatol 39, 3171- 3187. Online: https : / / doi . org / 10. 1002/joc.6009. 